\title{
Insect-Inspired Wing Actuation Structures Based on Ring-type Resonators
}

\author{
Caspar T. Bolsman ${ }^{a}$, Johannes F.L. Goosen ${ }^{a}$ and Fred van Keulen ${ }^{a}$ \\ ${ }^{a}$ Delft University of Technology, Faculty of Mechanical, Maritime and Materials Engineering, \\ Mekelweg 2, Delft, The Netherlands
}

\begin{abstract}
In this paper, we illustrate and study the opportunities of resonant ring type structures as wing actuation mechanisms for a flapping wing Micro Air Vehicle (MAV). Various design alternatives are presented and studied based on computational and physical models.

Insects provide an excellent source of inspiration for the development of the wing actuation mechanisms for flapping wing MAVs. The insect thorax is a structure which in essence provides a mechanism to couple the wing muscles to the wings while offering weight reduction through application of resonance, using tailored elasticity. The resonant properties of the thorax are a very effective way to reducing the power expenditure of wing movement. The wing movement itself is fairly complex and is guided by a set of control muscles and thoracic structures which are present in proximity of the wing root.

The development of flapping wing MAVs requires a move away from classical structures and actuators. The use of gears and rotational electric motors is hard to justify at the small scale. Resonant structures provide a large design freedom whilst also providing various options for actuation. The move away from deterministic mechanisms offers possibilities for mass reduction.
\end{abstract}

Keywords: Flapping wing, Micro Air Vehicle, Resonance, Compliant Mechanisms, Insects

\section{INTRODUCTION}

The design and development of flapping wing Micro Air Vehicles (MAV) requires the engineer to search for solutions outside the normal scope of flying crafts. Especially the quest for miniaturization of flapping wing MAVs forces an engineer to find inspiration by looking at flapping flight in nature. Insects, and to a lesser extent birds provide inspiration for many aspects of the development of flapping wing MAVs.

Flying insects have been, and are being, studied by many different scientific research disciplines. For the development of flapping wing MAVs the results of aerodynamic studies on flapping wings and flying insects are of key interest. ${ }^{1}$ These studies provide insight in the aerodynamic loads and energy cost of wing motion. The morphology and chemical functioning of tissues in the insect thorax have been extensively studied by mainly biologists. These studies have provided information on how insects create the power to move their wings and provide control. ${ }^{2,3}$ It has been shown that the insect thorax is a resonant system which drives the wings. This reduces the work to be delivered by actuators to accelerate and decelerate the wings and thus reduce the energy required for wing movement.

Since the recent renewed interest in flapping MAVs, many groups have contributed to the successful implementation of insect inspired resonant mechanisms to drive the flapping MAV wings. The developments in this paper are part of a larger project which aims at the development of a flapping wing MAV. The intended size is $10 \mathrm{~cm}$ wing span and $4 \mathrm{gr}$ vehicle mass. The targeted dimensions are feasible by using conventional technologies (gears, links and electric motors). ${ }^{4-7}$ These dimensions are also the starting point for other technologies (linear actuators and compliant mechanisms), ${ }^{8-11}$ which are more relevant when moving to even smaller dimensions.

Further author information: (Send correspondence to C.T. Bolsman)

C.T. Bolsman: E-mail: c.t.bolsman@tudelft.nl, Telephone: +31(0)152783522

Active and Passive Smart Structures and Integrated Systems 2008, edited by Mehdi Ahmadian Proc. of SPIE Vol. 6928, 69281I, (2008) -0277-786X/08/\$18 · doi: 10.1117/12.776084 
In the present setting there exist two types of flapping wing MAVs, with and without the ability to hover. Current focus is on a structure which provides the ability to hover. More specific, hovering flight should be the basic flying state. In insects and hovering birds the ability exists to reconfigure the wing movement and wing stroke plane to change between hovering and forward flight. Although possible, this is not aimed for in the current project. Hovering is the worst case scenario from an energy perspective, since no additional lift is created by induced flow by forward movement. Movement should result as deviations from the hovering state. Since the hovering state will be based on resonance, it will not be possible to introduce very large changes in the movement, consequently resulting velocities will be low. The developments described in this work are centered around the analysis and design of the use of a ring type structure as the basis for resonating wing actuation mechanisms.

\section{RESONANCE IN FLAPPING MICRO AIR VEHICLES}

The flying insect thorax is a complex mechanical structure that produces the wing motion that is needed to produce lift and thrust. The thorax structure is able to produce a complex 3 dimensional wing motion. ${ }^{12}$ The thorax structure is composed of a wing root joint, which is a complex multi Degree-of-freedom joint, the main driving muscles, which provide the power to overcome the aerodynamic drag and produce lift, the control muscles which are used to configure the wing root joint on a beat-to-beat basis, and finally, there is a set of elastic structures for energy storage. These structures include the thoracic parts, diverse ligaments, and the elastic properties of the main driving muscles. For an accurate description of insect physiology, see, for example, Chapman ${ }^{13}$ and Dudley. ${ }^{14}$

As described earlier, the resonant properties of the insect thorax lead to reduced energy expenditure for the basic movement of the wings. In its simplest form, the insect thorax system can be described by a one-

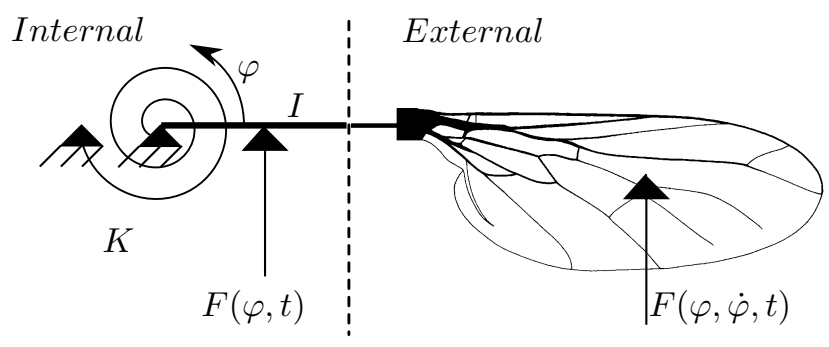

Figure 1: Systematic view of the resonant system

dimensional damped resonator, as can be seen in Fig. 1. This system can be described by the one-dimensional Euler equation of motion

$$
I \ddot{\varphi}+K \varphi=F(\varphi, t)+F(\varphi, \dot{\varphi}, t),
$$

Here, $I$ denotes the generalized inertia properties of the system and $K$ reflects the generalized stiffness properties of the system. $F(\varphi, \dot{\varphi}, t)$ is the time, velocity and position dependant generalized damping caused by the aerodynamics and $F(\varphi, t)$ is the generalized actuation force. Deformations are described by generalized displacement $\varphi$. In a resonant setting the deflection of the wing and thorax will reach their maximum at the same time. However, due to resonant amplification, the relation between the two might not be linear. This is important when using the energy approach, which is more interesting in this setting. Azuma, ${ }^{15}$ among others, state that when the insect thorax is driven in its natural frequency, the elastic and inertial torques cancel out and the following balance is left:

$$
\int_{t}^{t+T} F_{\text {aero }} d \varphi+\int_{t}^{t+T} F_{\text {actuators }} \cdot d \varphi=0
$$

This assumes that the only dissipation per cycle is aerodynamic dissipation, and losses due to friction and material hysteresis cycles have been ignored. The most important feature of resonance in the flapping setting is amplitude amplification of the wing stroke. It should be noted that in this one-dimensional approach the aerodynamics is simplified to just aerodynamic damping on the wing. This simplification implies that there is no knowledge on the magnitude or efficiency of lift production. In order to include knowledge on lift generation there 
are two options. First, the use of full-detail CFD models ${ }^{16}$ and, second, the use of quasi-steady aerodynamic models. ${ }^{17,18}$ Of which the latter is currently the only viable solution in this exploratory design setting.

Thus, the intent of employing resonance in the design of the wing-actuation mechanism of flapping wing MAVs is to reduce the actuator power needed to accelerate and decelerate the wings in the reciprocal wing movement. This property has been extensively studied in insects and birds. ${ }^{19}$ From Ennos ${ }^{20}$ it can be seen that inertial loads dominate the aerodynamic loads. This observation is supported by research on the quality of resonance the insect thorax system, ${ }^{21}$ which is generally high for resonant aerodynamically damped biological systems such as the insect thorax. Both these facts lead to the conclusion that it is energy wise not interesting to vary wing beat frequency in insects and flapping MAVs, due to rapidly decreasing resonant response when moving away from a resonant frequency.

Summarizing; the insect thorax is a large deflection high efficiency resonator which controls energy flows from muscles to aerodynamic dissipation. A technical equivalent of the described thorax is a good design alternative for an insect scale flying robot.

\section{RING-BASED RESONATORS}

The design and development of flapping wing MAVs requires the engineer to re-explore the design space that is already filled with successful designs by nature. Centering around the thorax section, an engineering alternative has to be found for sustaining flapping motion while also converting mechanical energy into lift forces. This is one part of the requirements. The other is the required mechanical amplification of the actuator stroke. Generally speaking, the effective actuation strain of linear actuators suitable for application in resonance driven flapping MAVs, such as electromagnetic, piezo electric and electroactive polymers, is not very large. ${ }^{22}$ Due to the large differences between actuator strain and required wing deflection the need exists for motion amplification. This can be achieved through mechanical amplification and by the use of properly chosen flexibility to exploit resonance. A Combination seems particularly interesting in this setting.

Because of stringent weight constraints, an alternative has to be found to store strain energy in a more distributed sense. The available mechanical deformation modes, extension, compression, torsion, shear and bending, are all suitable to store energy. Bending can be used in a self-contained setting, namely a ring-type structure. The ring studied here is built from strip-like material, i.e. thickness is very small compared to width, which is shaped into circle. The inertia, stiffness and dimensions of the ring can be used to tune the whole wing actuation mechanism for resonance at a specific frequency with specific amplitude amplification. For the ring, bending modes are reviewed in the current setting, as generally the torsional modes result in much higher eigenfrequencies. Analytical solutions exist for vibrating rings ${ }^{23}$ including both bending and torsional modes. These solutions are not used in this setting due to rapidly increasing complexity when adding different actuator and damping models and other mechanics.

The benefits of using rings in the current setting are, the freedom in wing coupling options, absence of the need for a support structure, and the center of the ring offers room to place the actuator. One of the major drawbacks is coupled to a benefit. Since there are no supports structures difficulties will rise when in a later stage electronics and power source have to be coupled to the flapping wing MAV. Especially sensors might be negatively influenced by the vibrating movement.

\section{MODELING OF RING-TYPE STRUCTURES}

Based on the ring concept various options exist to mount the wings.As the deformation of the ring is small when compared to deflections of the wing, the way the wings are kinematically connected to the ring determines the amplitude amplification which can be reached. Two different connections can be distinguished The connection can be very compliant, implying the wings can be seen as a deforming part of the resonating structure and thereby provide amplitude amplification. In a pseudo-rigid-body setting, comparatively rigid structures are coupled by elastic hinges, implying the structure uses mechanical amplification to provide amplitude amplification.

In this study, four structures are proposed to study possibilities of the applications of ring type resonators. These structures are studied first numerically, later they are realized and tested. The 4 designs are explained 
in subsections 4.1, 4.2, 4.3 and 4.4. Since the analysis track is identical for all four structures, only a general description of the analysis is given in subsection 4.5 .

\subsection{Design 1}

The first design is the simplest possible extension of the ring to include flapping wings. The wings are formed by a bending beam, which is rigidly coupled to the ring at the center position. As described earlier the wings are a deforming part of the structure in this design. The main mode of amplitude amplification is the bending of the wings. This feature is not found in insects but still interesting to test as an engineering solution. The structure is driven by a linear actuator inside the ring, which is coupled to the ring in such a way that its direction of actuation is perpendicular to the wingspan. The design is shown in Fig. 2a. The multi body equivalent model is shown in Fig. $2 \mathrm{~b}$

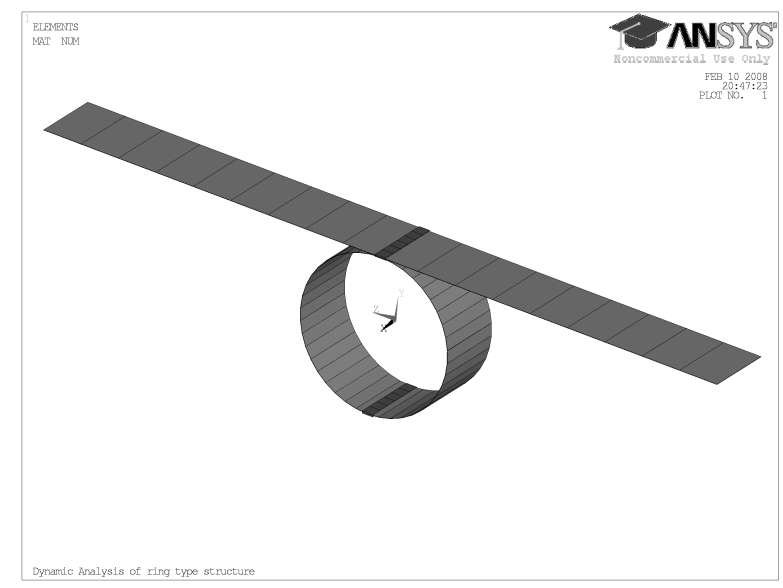

(a) Two compliant wings coupled to the ring

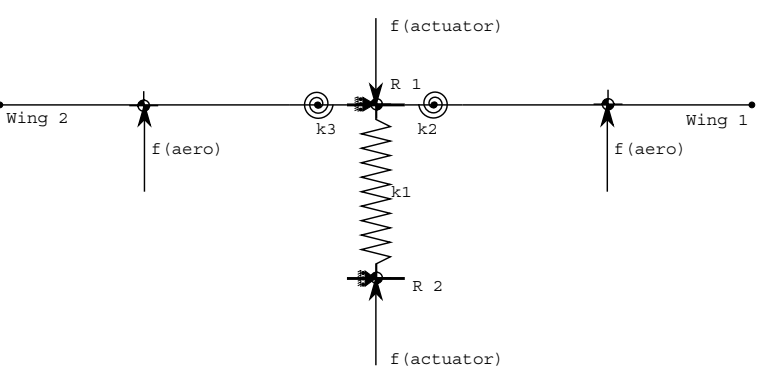

(b) Pseudo-rigid-body model for Design 1

Figure 2: Design 1: Flexible wings are the main mechanism for amplitude amplification.

\subsection{Design 2}

The second design exploits a compliant mechanism for mechanical amplification. The wings are elastically connected to a side of the ring. Struts are in place, which connect the opposite side of the ring to the wing. By these means the linear motion of the ring is converted into a large rotation at the wing base. The wing connection setup is inspired by the work done by Cox et al. ${ }^{8}$ All compliant connections are flexure hinges. The actuator is connected in the same manner as described in subsection 4.1. The design is shown in Fig. 3a. The main mode of amplitude amplification is now carried by the compliant mechanism, note that the wings do not store elastic energy in this design, as they are modeled as being rigid. Along the wingspan this is a valid assumption. Torsional deformation has to be modeled when lift production is studied. This design is better suited for a pseudo-rigid-body representation, see Fig. 3b.

\subsection{Design 3}

The third design is, in essence, an extension of the second model. The number of wings is doubled to four. A major benefit of this setup is the positioning of the wings. Their stroke plane, as compared to the center of mass, is far better suited to support hovering flight. The struts now interfere with each other, meaning that the realization has to include a small offset. The model is shown in Fig. 4a and the pseudo-rigid-body representation is shown in Fig. 4b. 


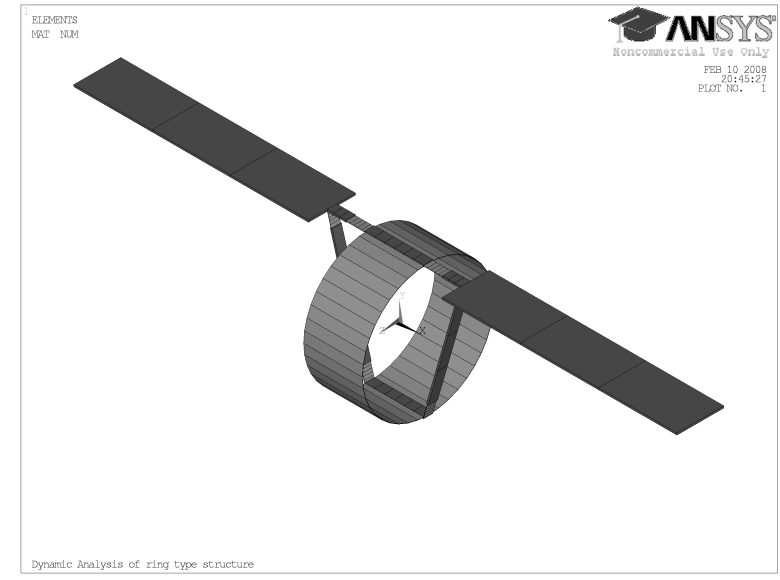

(a) Two wings coupled to the ring by a compliant mechanism

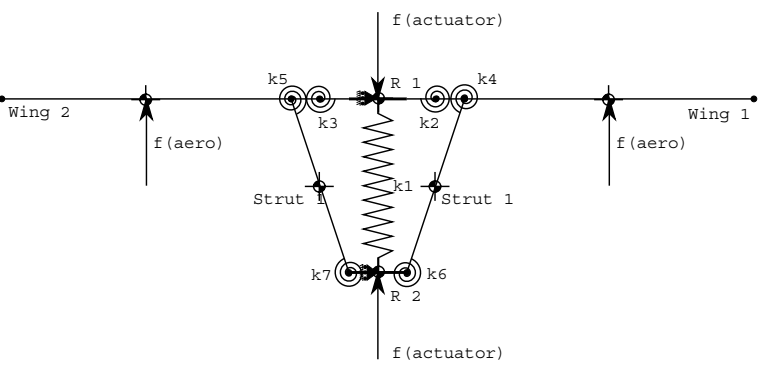

(b) Pseudo-rigid-body model for Design 2

Figure 3: Design 2: Mechanical amplification as the main amplitude amplification source.
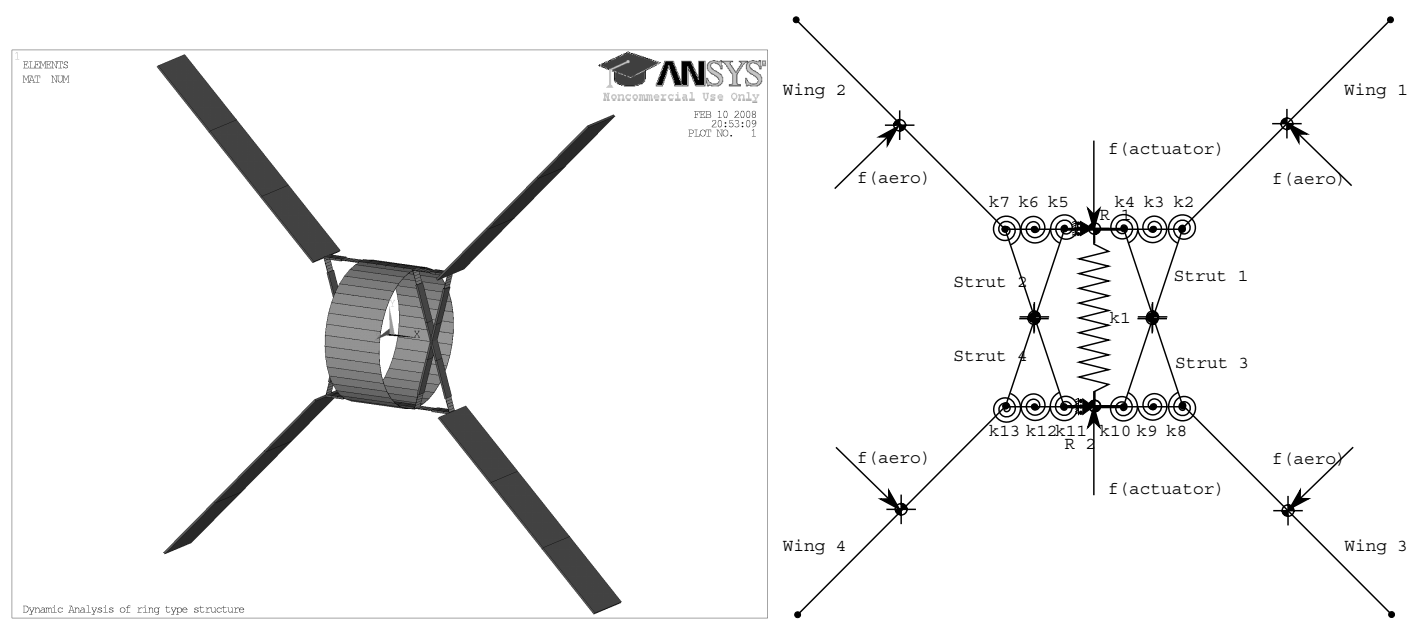

(a) Four wings coupled to the ring by a compliant(b) Pseudo-rigid-body model for Design 3 mechanism

Figure 4: Design 3: Mechanical amplification as the main amplitude amplification source. Extension of Design 2 .

\subsection{Design 4}

The fourth design is a simplification of the third, that is, two struts have been removed. The side of the remaining struts which was coupled to the ring is now coupled to the wing at the same position as the other side. This is primarily for weight reduction. The benefits with respect to the orientation of the stroke plane are also valid for this setup. The design is shown in Fig. 5a, whereas the pseudo-rigid-body representation is depicted in Fig. 5b.

\subsection{Numerical Modeling}

The numerical modeling of the ring-based structures is composed of two parts. The first is a Multi-Body Dynamics (MBD) analysis, the second is Finite Element (FE) analysis.

The first analysis relies on a 2-dimensional MBD representation, which is numerically analyzed with Matlab ${ }^{\circledR}$. The chosen approach is Newton-Euler with constraints. It is used to gain insight in the kinematics of the 


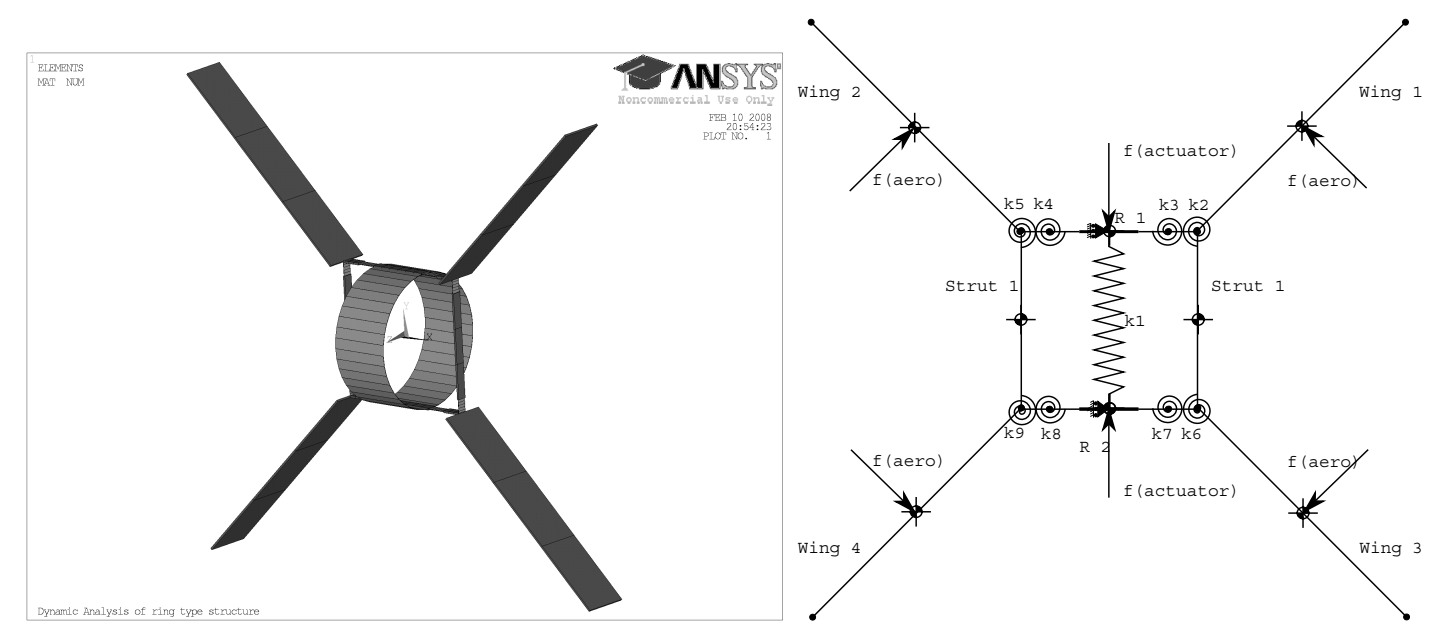

(a) Four wings coupled to the ring by a compliant (b) Pseudo-rigid-body model for Design 4 mechanism

Figure 5: Design 4: Mechanical amplification as the main amplitude amplification source. Simplification of Design 3.

models and to search for the correct sizing parameters. Both actuation and aerodynamic damping are included. Actuation is implemented by applying a sinusoidal force on the intended position of the actuator. Of the aerodynamics only the drag component is included, whereas the lift components are not included. The lift, and effectively also the drag, are dependent on the wing kinematics. In order to accurately model lift production a quasi-steady aerodynamics model has to be included. ${ }^{24}$ The drag is implemented as viscous damping applied on the wings in the effective center of pressure, this approach is suited for the current exploratory setting and used by Tantanawat and Kota ${ }^{25}$ among others. The elastic hinges are implemented using equivalent elastic models based on pseudo-rigid-body theory. ${ }^{26}$ The multi body representation of the four models is depicted in Fig. 2b, Fig. 3b, Fig. 4b and Fig. 5b.

Besides the aforementioned assumptions with respect to actuation and aerodynamics, there have been assumptions on the representation of the mass and stiffness properties of the ring. The elastic behavior of the ring, seen from the wing root, has been assumed to be linear as small deflections of the ring are anticipated. Therefore the elastic properties can be represented by a linear spring. The mass distribution of the ring is assumed lumped in bodies $R 1$ and $R 2$ for all models. The assumption of small displacements is used here again. The mass of the actuator acts on bodies $R 1$ and $R 2$ meaning that the mass of the actuator is also lumped here. The actuator mass is much larger than the mass of the ring indicating that the interpolation function chosen to lump the mass of the ring has a small influence on the results.

Time integration of the equations of motion is performed to simulate responses. As mentioned, the multi-body models are primarily used for sizing the elastic hinges and the topology of the structure.

The FE-models are parametrized to easily adjust to parameter changes. Parameters from the multi body analysis are thus easily implemented. The structures are composed of strips and struts and elastic hinges. Two options exist for modeling these structures, shell and beam elements. Beams are chosen to discretize the structures. Beams are well suited for representing the bending aspects of the anticipated deformations, and although shells are the obvious choice for the wings, as no wing deformation of the wing membrane has to be modeled beams are also a valid choice for the wings.

The damping is implemented by applying rotational viscous dampers at the wing root, This assumption is valid for Designs 2, 3 and 4. which have relatively stiff wings. For Design 1 linear dampers have been used which attach to the aerodynamic center of pressure. Actuation is implemented by applying sinusoidal force on the intended position of the actuator, similar to the MBD analysis. 
Boundary conditions are applied such that they accurately represent the conditions in free flight. The material properties and topology used represent the real material properties and topology which are later used to realize the structures. First a modal analysis is performed to find the eigenfrequencies of the designs. The only eigenfrequency of interest, at this time, is the one that corresponds to flapping wing flight. These frequencies are used as a starting point in a nonlinear transient analysis to simulate the responses due to the actuation at resonance. The eigenfrequency is a valid starting point in the nonlinear analysis. Two factors exist which influence the resonant response when deflections become large. First, geometrically nonlinear behavior and second, the magnitude of the damping. Implicitly small damping has been assumed. The FE-analysis is performed to gain information on how the structures react at large deflection resonance subject to designs found by the MBD analysis.

\section{REALIZATION}

In order to review the value of the predictive capabilities of modeling methods presented and to view how the concepts perform, prototype structures have been built and tested in a laboratory setup.

The first part is the realization of the models. The main part of the designs is the ring. Different materials have been reviewed for the ring. The material has to have excellent elastic properties and low hysteresis. For the current exploratory setting ease of handling is also an issue. Spring steel has excellent elastic properties and is easy to handle. However, materials exist which have higher specific strain energy density, which will become important when mass constraints are more stringent. The same steel is suitable for the construction of the flexure hinges. The struts are unidirectional carbon fiber beams for light weight, stiffness is not an issue due to limited loads. The same carbon fiber beams are used for the wing spar. Plastic sheet is used to create wing surface for aerodynamic damping. The present wings are too stiff to deform enough for efficient lift production. The models are shown in Fig. 6 and Fig. 7. The actuation is done by solenoid actuators. These actuators are

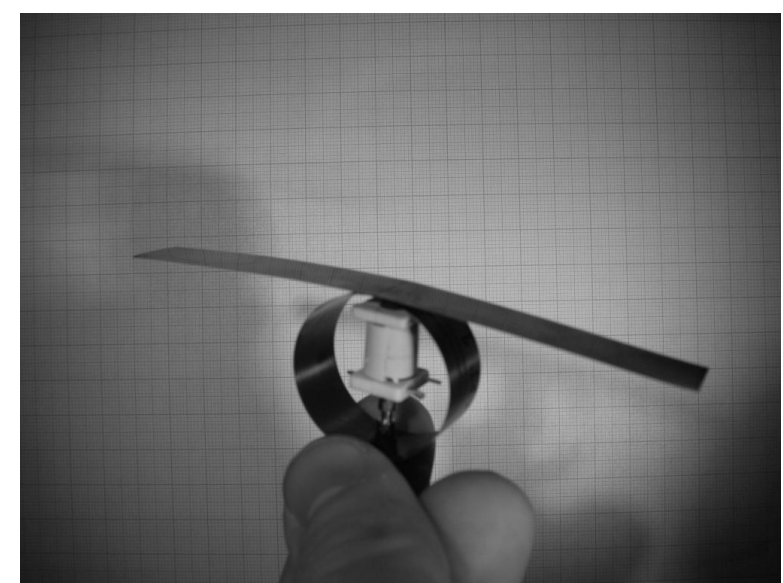

(a) Two winged design amplitude amplification by(b) resonance of compliant structure.

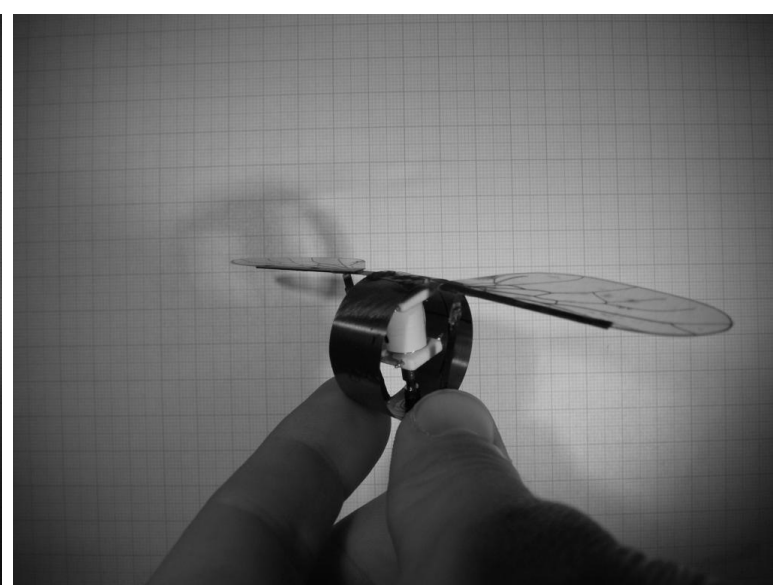

(b) Two winged design amplitude amplification based on compliant mechanism.

Figure 6: Two winged designs 1 and 2.

modified off-the-shelf actuators. The solenoids are positioned in the center of the ring. A frequency generator with amplifier is used to drive the solenoids.

The prototypes are tested in a laboratory setup. In this setup the prototypes are suspended in a small studio in order to make photographs of the actuated models, the suspension is such that it represents as good as possible the conditions in free flight.

Visual inspection is used to estimate the maximum deflection of the wings to search for resonance. This process is supported and guided by results from the numerical modeling. As in the numerical modeling, only 


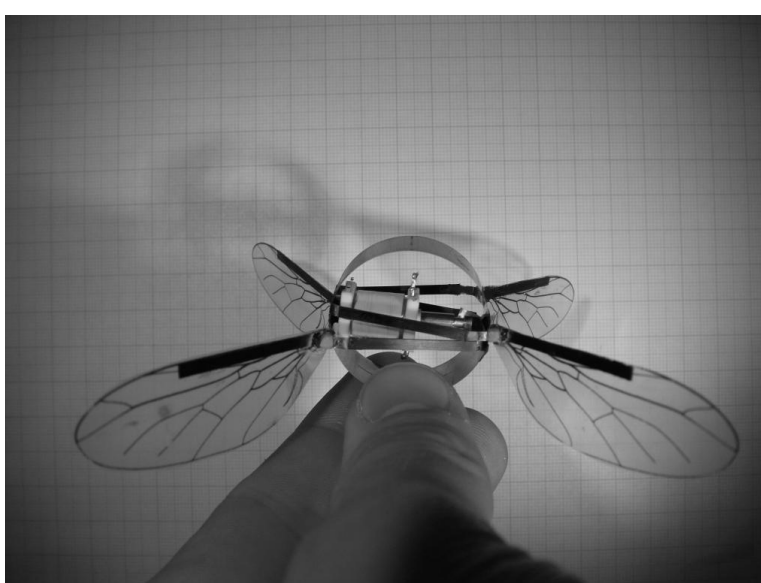

(a) Four winged design, extension of design 2

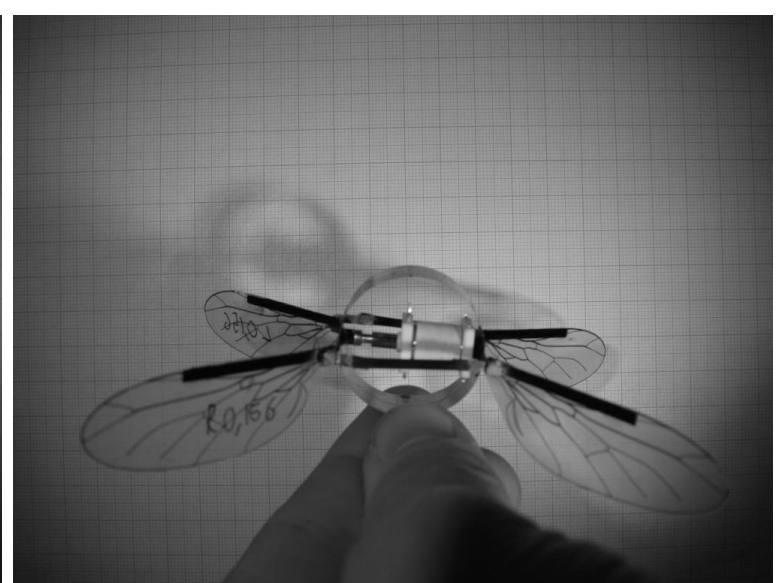

(b) Four winged design, simplification of design 3

Figure 7: Four winged designs 3 and 4.

the eigenfrequency that corresponds to flapping flight is of interest, this is not necessarily the first eigenfrequency. Photographs are made using shutter time which is significantly larger than the cycle time at resonance. Amplitudes can be estimated from the photographs.

\section{RESULTS}

The results of the numerical modeling, finite element and multibody dynamics, and the testing of the prototypes are all important for the review of the designs. Early indications from results of Design 1 indicate that this idea is not able to convey enough power from the actuator to the wings while maintaining a large amplitude wing stroke. The predicted eigenfreqencies are very low, using the current actuation and realization, the models could not produce large deflections. We will therefore focus on Designs 2, 3 and 4.

The results of the transient analysis in the FE-models shows that the designs are able to produce large deflections, whilst being actuated at resonance. Large amplitude deflections of the models are shown in Fig. $8 \mathrm{a}$, b and c., superimposed on the undeformed configuration, for Designs 2, 3 and 4 respectively. The start up

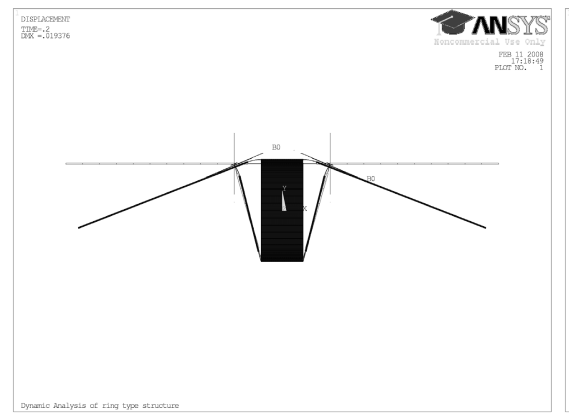

(a) Design 2

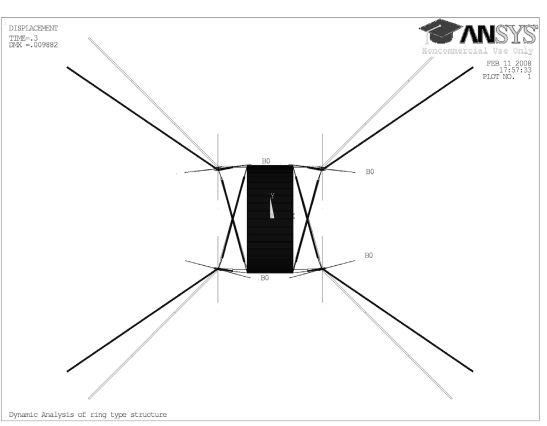

(b) Design 3

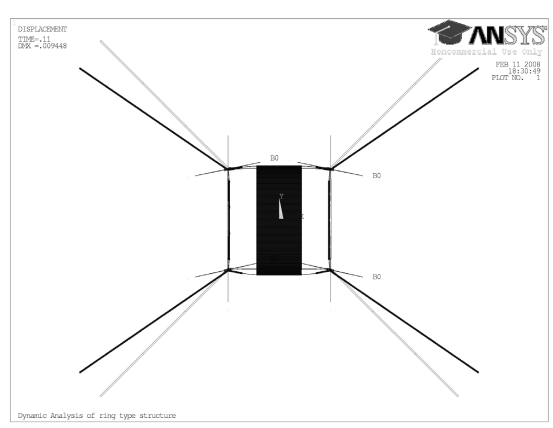

(c) Design 4

Figure 8: Maximum amplitude deflection for Designs 2, 3 and 4.

performance of the designs is plotted in Fig. 9. In this figure the first 0.5 seconds of actuation is plotted starting from the rest position. Rotational deflection of the wing root is plotted for each tested design. Using the described laboratory setup the models can be measured. The designs are actuated at their resonant frequency. This frequency has been previously found by sweeping through the expected range around the intended eigenfrequency 


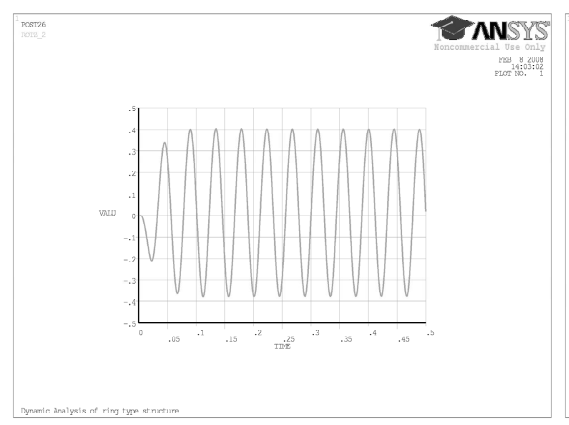

(a) Design 2

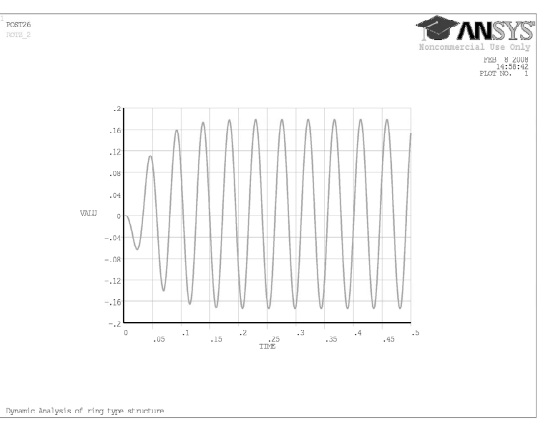

(b) Design 3

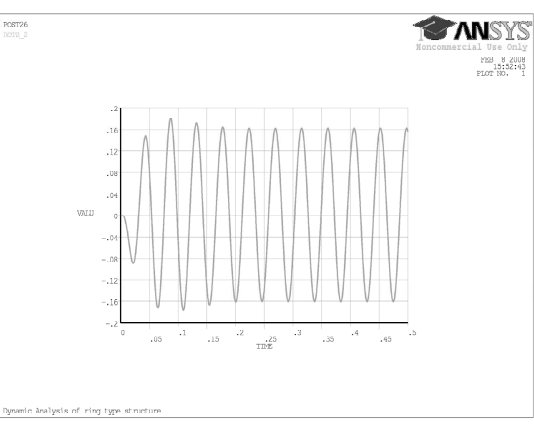

(c) Design 4

Figure 9: Wing root rotation $[\mathrm{rad}]$ for Designs 2, 3 and 4.

from the FE-analysis. Photographs of the Designs,actuated at resonance, are shown in Fig. 10a, b and c. for Designs 2, 3 and 4 respectively. Both the results from the numerical modeling and the realized designs are

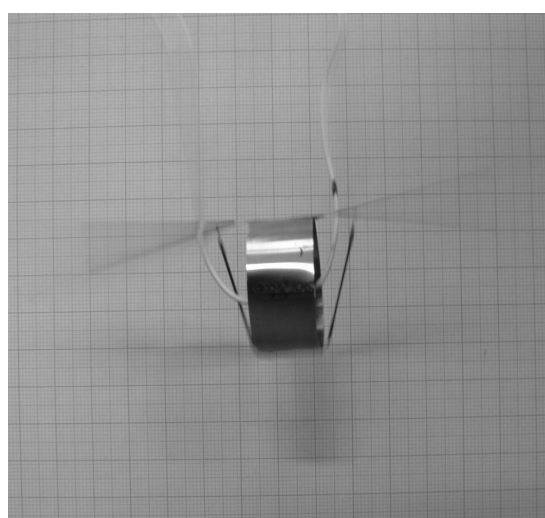

(a) Design 2

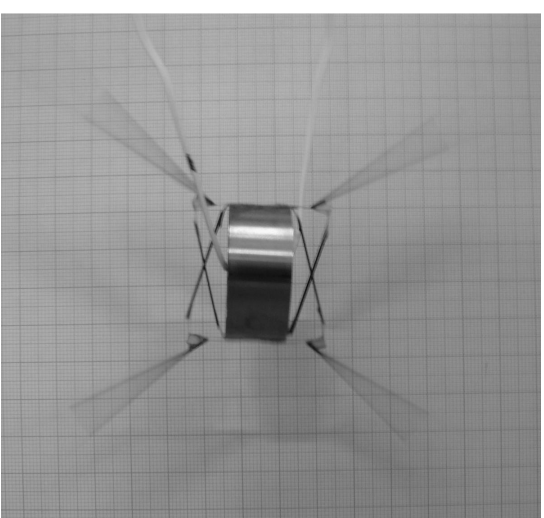

(b) Design 3

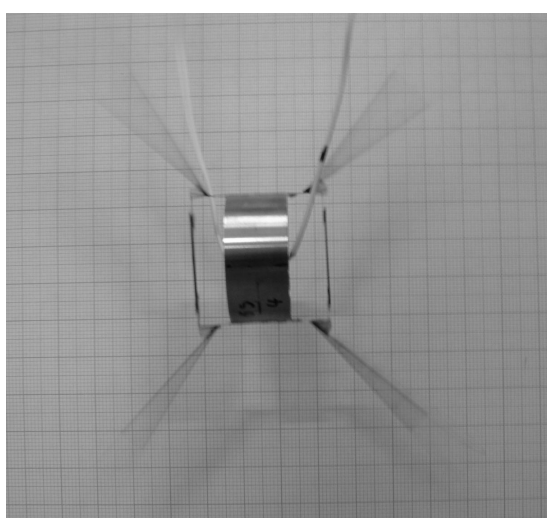

(c) Design 4

Figure 10: Realized models actuated at resonance

encouraging for the successful use of rings as the main energy storage in flapping wing MAVs. There are differences between the FE models and the laboratory tests. First, large deflections are easily obtained by the FE models. For the laboratory tests it proved more difficult to obtain large amplitude amplification. Second, the eigenfrequencies from the FE models and the test are given in Table 1, which shows the difference between the two. Large differences exist, but these results still show for the predictive value of the FE modeling. These differences arise from both modeling errors and errors in the realization. The startup performance of the FE models shows that within 0.1 seconds the models reach their maximum deflection.

Design 1 should be studied more to investigate other configurations and parameters which might improve performance. Design 2 provides enough deflection to drive flapping wings. Aim here is at hovering flight, which makes this design less suitable. The positioning of the wings makes it difficult to have a horizontal flapping plane. This problem does not exist for Designs 3 and 4. The configuration with four wings is ideal for a hovering platform. Both designs are able to function with large amplitude deflections. The differences in strut topology makes their functioning different. When general dimensions are the same, then the wings in Design 4 move half that of those in Design 3 with identical ring deformation. They are both usable when dimensions, of hinges and struts and their attachment points are further optimized. FE simulations have shown that Design 4 has eigenmodes around the main flapping eigenmode which might be interesting for control purposes, this feature is open for further study. 
Table 1: Comparison of the numerical test and results from the laboratory testing

\begin{tabular}{|l|l|l|l|}
\hline Table & Model 2 & Model 3 & Model 4 \\
\hline Eigenfrequency FE model $(\mathrm{Hz})$ & 22.58 & 21.88 & 26.26 \\
\hline Eigenfrequency Test Model(Hz) & 26 & 28 & 24 \\
\hline Difference (\%) & $\sim 15 \%$ & $\sim 28 \%$ & $\sim 9 \%$ \\
\hline
\end{tabular}

\section{DISCUSSION}

It can be concluded that both the MBD approach and the FE approach, are suited for the analysis and pre-design of the structures studied here. Depending on the type of information required, a choice between the two has to be made. The MBD approach is more suited for parameter estimations. Its strength is in the low number of degrees of freedom and thus computational efficiency. The nonlinear transient FE-analysis is well suited for analysis of compliance and compliant structures for resonant systems.

As can be seen from the results, there is a mismatch between computational and tested eigenfrequencies. This has two main causes. First, the manufacturing tolerances are very high in the current prototyping setting. This is especially true for the flexure hinges.

The representation of free flight conditions in the numerical model does more accurately represent real free flight conditions than the suspension in the laboratory tests. The lead wires, which are relatively stiff compared to the involved forces, introduce disturbances in the mass and stiffness distribution of the system. For further testing different, more compliant and lightweight, mounting methods have to be introduced. In order to get better correspondence the models should be adjusted to include the suspension.

The use of solenoids is well suited for the current exploratory setting. Off-the-shelf actuators carry much excess mass and the low specific power of electromagnetic actuators in general, is not suited for low mass applications such as in MAVs. For real flight at this scale custom electromagnetic actuators could provide enough power. When scaling down the engineer should look at other actuator technologies due to less favorable scaling of electromagnetic actuators.

The wings in these designs are relatively small as compared to the body. The wings already have the intended dimensions, the body needs to be scaled down. For the ring scaling laws do not introduce great problems, since there are many design parameters which can be used to tune the system. Material thickness, ring radius and ring width.

The wings used are too stiff to be deformed by the applied aerodynamic forces. In order to produce lift wings have to be attached, which have a tailored stiffness. In larger insects, i.e. with a size comparable with the present design, there is considerable torsional deformation of the wings. Bending of the wing in chord direction or along the span is small. In insects a large part of wing rotation has its source in the compliance of the wing joint. In flapping wing MAVs a similar system has to be introduced, a combination of wing torsion and compliant hinge at the wing root.

\section{CONCLUSION}

The application of ring type structures as the basis for flapping MAVs has been researched at an exploratory level in this paper. The observations indicate that the ring is suitable to be used as the main elastic energy storage unit. The use of flexure hinges to amplify the resonant motion is suitable and interesting. The topologies of structures provide good results but materials and dimensions have to be tuned further.

Both the multi-body based and finite element modeling provide results which help the engineer to come up with valuable designs. The methods provide useful tools for either rapid development parameter estimates or accurate simulation of time response. Especially the finite element model should be extended to include a more accurate model for aerodynamics and also the wing. 
The 4 winged concepts are deemed most promising due to the fact that the flapping plane is horizontal. Due to symmetric movement of the wings the body will stay relatively still, which benefits possible sensors. When the center of mass is placed below the vertical position of the flapping plane pendulum stability can be induced to reduce the need for control.

\section{ACKNOWLEDGMENTS}

This work is performed within the setting of a cooperation between DevLab and the Delft University of Technology. This work was supported by the DevClub and Casimir/NWO as part of the Atalanta project within DevLab. The authors would like to thank Wim Hendriksen from the DevLab for his helpful discussions and useful input.

\section{REFERENCES}

1. S. A. Ansari, R. Żbikowski, and K. Knowles, "Aerodynamic modelling of insect-like flapping flight for micro air vehicles," Progress in Aerospace Sciences 42, pp. 129-172, February 2006.

2. C. H. Greenewalt, "The wings of insects and birds as mechanical oscillators," Proceedings of the American Philosophical Society 104, pp. 605-611, December 1960.

3. F. O. Lehmann and M. H. Dickinson, "The control of wing kinematics and flight forces in fruit flies (drosophila spp.)," The Journal of Experimental Biology 201, pp. 385-401, 1998.

4. C. Galinski and R. Żbikowski, "Insect-like flapping wing mechanism based on a double spherical scotch yoke," Journal of the Royal Society: Interface 2, pp. 223-235, June 2005.

5. C. Galinski and R. Żbikowski, "Materials challenges in the design of an insect-like flapping wing mechanism based on a four-bar linkage," Materials \&5 Design, 2006.

6. V. Malolan, M. Dineshkumar, and V. Baskar, "Design and development of flapping wing micro air vehicle." 42nd AIAA Aerospace Sciences Meeting and Exhibit, January 2004.

7. M. T. Keennon and J. M. Grasmeyer, "Development of the black widow and microbat mavs and a vision of the future of mav design." AIAA/ICAS International Air and Space Symposium and Exposition: The Next 100 Years, July 2003.

8. A. Adam Cox, D. Monopoli, D. Cveticanin, M. Goldfarb, and E. Garcia, "The development of elastodynamic components for piezoelectrically actuated flapping micro-air vehicles," Journal of Intelligent Material Systems and Structures 13, pp. 611-615, September 2002.

9. J. Yan, R. Wood, S. Avadhanula, M. Sitti, and R. S. Fearing, "Towards flapping wing control for a micromechanical flying insect." IEEE International Conference on Robotics and Automation, pp. 3901-3908, Seoul, Korea, May 2001.

10. X. Deng, L. Schenato, W. C. Wu, and S. Sastry, "Flapping flight for biomimetic robotic insects - part i: System modeling," Tech. Rep. UCB/ERL M04/35, EECS Department, University of California, Berkeley, 2004.

11. X. Deng, L. Schenato, and S. Sastry, "Flapping flight for biomimetic robotic insects - part ii: Flight control design," Tech. Rep. UCB/ERL M04/34, EECS Department, University of California, Berkeley, 2004.

12. F. O. Lehmann, "Aerial locomotion in flies and robots: Kinematic control and aerodynamics of oscillating wings," Arthropod Structure \& Development 33, pp. 331-345, 2004.

13. R. F. Chapman, The Insects Structure and Function, The English Universities Press LTD, 1969.

14. R. Dudley, The biomechanics of insect flight: form, function, evolution, Princeton University Press, 2000.

15. A. Azuma, The Biokinetics of Flying and Swimming, American Institute of Aeronautics and Astronautics, Inc., 2006.

16. H. Liu, C. P. Ellington, K. Kawachi, C. van den Berg, and A. P. Willmott, "A computational fluid dynamic study of hawkmoth hovering," The Journal of Experimental Biology 201, pp. 461-477, 1998.

17. C. P. Ellington, "The aerodynamics of hovering insect flight. i. the quasi-steady analysis," Royal Society of London Philosophical Transactions Series B 305, pp. 1-15, feb 1984.

18. S. P. Sane and M. H. Dickinson, "The aerodynamic effects of wing rotation and a revised quasi-steadi model of flapping flight," The Journal of Experimental Biology 205, pp. 1087-1096, 2002. 
19. C. P. Ellington, "The aerodynamics of hovering insect flight. v. lift and power requirements," Royal Society of London Philosophical Transactions Series B 305, pp. 145-181, feb 1984.

20. A. R. Ennos, "Inertial and aerodynamic torques on the wings of diptera in flight," The Journal of Experimental Biology 142, pp. 87-95, 1989.

21. C. P. Ellington, "The novel aerodynamics of insect flight: Application to micro-air vehicles," The Journal of Experimental Biology 202, pp. 3439-3448, 1999.

22. Y. Bar-Cohen, ed., Electroactive Polymer (EAP) Actuators as Artificial Muscles: Reality, Potential, and Challenges, SPIE-The International Society for Optical Engineering, 2nd ed., March 2004.

23. S. S. Rao, Vibration of continuous systems, Wiley, 2007.

24. B. Pålsson, "Overview of insect flight mechanics \& principal design of flapping wing actuation with actuator overview," Master's thesis, Chalmers University of Technology, 2006.

25. T. Tantanawat and S. Kota, "Design of compliant mechanisms for minimizing input power in dynamic applications." Proceedings of DETC 2006, International Design Engineering Technical Conference, September 2006.

26. L. L. Howell, Compliant Mechanisms, Wiley, 2001. 